\title{
Perspective Matching Using the EM Algorithm
}

\author{
A.D.J. Cross and E.R. Hancock \\ Department of Computer Science, \\ University of York, \\ York, Y01 5DD, UK. \\ erh@minster.york.ac.uk
}

\begin{abstract}
This paper describes a new approach to perspective matching which simultaneously exploits both rigidity-structure and point distribution information. The structural component of the model is represented by a Delaunay triangulation of the point-set. The point-distribution model is represented by a perspective deformation of the point-set. Modelmatching is realised using a variant of the EM algorithm. This involves coupling the correspondence matching of the Delaunay triangulation to the recovery of the point deformation parameters. We use a Bayesian consistency measure to gauge the relational structure of the point correspondences. Maximum-likelihood point deformation parameters are estimated using a mixture-model defined over the point error-residuals. In effect, the Bayesian consistency measure is used to weight the contributions to a mean-squares error-criterion. The method is evaluated on matching $2 \mathrm{D}$ objects under varying pose.
\end{abstract}

\section{Introduction}

Rigidity constraints play an important role in correspondence matching [6,11]. It was Ullman [11] who first suggested the use of point-rigidity as a way of imposing proximity constraints on point-sets. Several authors have drawn inspiration from Ullman's ideas in developing general purpose correspondence matching algorithms using the the Gaussian weighted proximity matrix. There are two contrasting uses of the proximity-matrix which deserve special mention. Scott and Lonquet-Higgins [7] locate correspondences by finding a singular value decomposition of the inter-image proximity matrix. Shapiro of Brady [9], on the other hand, match by comparing the modal eignestructure of the intra-image proximity matrix. In fact, these techniques provide the basic ground-work on which the deformable shape models of Cootes et al [3] and Sclaroff and Pentland [8] build. More recently, McReynolds and Lowe [6] have shown how rigidity constraints can be incorporated into the recovery of perspective correspondence matches. This algorithm uses Levenberq-Marquardt optimisation to locate least-squares correspondence matches.

Our aim in this paper is to present a statistical framework that allows both the rigidity structure and deformation properties of point-sets to be utilised in perspective matching. These two aspects of the matching process have distinct 
representations. Point-set deformation is modelled using a Gaussian mixturemodel for the co-ordinate error-residuals under a perspective transformation. Rigidity-structure is represented by a Delaunay triangulation [1] over the point sets being matched. We recover the correspondence matches using a variant of the EM algorithm. This is an iterative process which alternates between transformation parameter estimation in the maximisation step and updating matching probabilities in the expectation step.

Maximisation returns three pieces of information. The first of these is a set maximum-likelihood perspective parameters which are used to project the model-points onto the view-space of the data. The second piece of information is the variance-covariance matrix for the co-ordinate error residuals. Finally, we estimate a set of maximum a posteriori probability point correspondence matches between the nodes of the Delaunay triangulations representing the structure of the point-sets. In other words, the M-step models the co-ordinate projection of the deformed shape into a view space where measurements are taken. Expectation involves updating probability distributions which describe the current state of the matching process. Here we dichotomise between the point-distribution and structural aspects of our model.

The outline of this paper is as follows. In Section 2, we detail the elements of our point-matching process. Section 3 describes how we establish Delaunay graphs and exploit graph structure in finding correspondence matches. The twostep iterative matching process is detailed in Section 4 . Section 5 presents some experiments concerned with matching different 3D poses of planar shapes. Finally, Section 6 offers some conclusions and describes our future plans.

\section{Point Deformation}

In the work described here we are interested in matching 2D shapes under different perspective viewing conditions. Projective deformation of a point-set involves translation, rotation, affine shear and foreshortening. Our basic aim is to recover the parameters of the perspective transformation which brings a set of model or fiducial points into correspondence with their counterparts in a set of image data. Each point in the image data is represented by an augmented vector of co-ordinates $\underline{w}_{i}=\left(x_{i}, y_{i}, 1\right)^{T}$ where $i$ is the point index. The available set of image points is denoted by $\mathbf{w}=\left\{\underline{w}_{i}, \forall i \in \mathcal{D}\right\}$ where $\mathcal{D}$ is the point index-set. The fiducial points constituting the model are similarly represented by the set $\mathbf{z}=\left\{\underline{z}_{j}, \forall j \in \mathcal{M}\right\}$. Here $\mathcal{M}$ is the index-set for the model feature-points and the $\underline{z}_{j}$ represent the corresponding image co-ordinates. The aim of our matching algorithm is to iteratively recover a parameter-matrix $\Phi^{(n)}$ which describes the perspective co-ordinate transformation that brings the feature points in image and model into registration with one-another. The transformed co-ordinate vectors are computed in the following way

$$
\underline{\mathbf{z}}_{j}^{(n)}=\frac{1}{\underline{\mathbf{z}}_{j}^{T}, \Psi^{(n)}} \Phi^{(n)} \underline{\mathbf{z}}_{j}
$$


In the above equation the matrix $\Phi^{(n)}$ models the perspective transformation of co-ordinates. In the work reported here we are not concerned with explicitly recovering the geometry of translation, rotation, affine shear and foreshortening. Instead, we adopt the following parameterisation of the perspective deformation of the point-set. The required transformation matrix has nine free parameters and is of the form

$$
\Phi^{(n)}=\left(\begin{array}{lll}
\phi_{1,1}^{(n)} & \phi_{1,2}^{(n)} & \phi_{1,3}^{(n)} \\
\phi_{2,1}^{(n)} & \phi_{2,2}^{(n)} & \phi_{2,3}^{(n)} \\
\phi_{3,1}^{(n)} & \phi_{3,2}^{(n)} & \phi_{3,3}^{(n)}
\end{array}\right)
$$

The superscript on the matrix indicates that the parameters are taken at $n^{\text {th }}$ iteration of our algorithm. The vector $\Psi^{(n)}=\left(\phi_{3,1}^{(n)}, \phi_{3,2}^{(n)}, 1\right)^{T}$ is formed from the elements of the bottom row of the perspective parameter matrix. Expressed in this form, the transformation matrix subsumes the simpler Euclidean and affine cases which require four and six parameters respectively.

\section{Structural Deformation}

One of our goals in this paper is to exploit structural constraints to improve the recovery of perspective parameters from sets of feature points. Because of its well documented robustness to noise and change of viewpoint, we adopt the Delaunay graph as our basic representation of image structure [1]. We establish Delaunay graph representations of data and model, by seeding Voronoi tessellations from the feature-points. Our aim is to exploit the structure of the Delaunay graphs to impose constraints on the recovery of perspective parameters. The overall parameter estimation strategy is based on the expectation-maximisation algorithm. We incorporate the structural information into the parameter estimation process by borrowing an idea from the hierarchical mixture of experts algorithm of Jordan and Jacobs [5]. We use the probabilities of structural matches to gate the expected likelihood function. These gating probabilities are computed using the framework for relational graph matching recently reported by Wilson and Hancock $[13,14,12]$.

The process of Delaunay triangulation generates relational graphs from the two sets of point-features. More formally, the point-sets are the nodes of the a data graph $G_{D}=\left\{\mathcal{D}, E_{D}\right\}$ and a model graph $G_{M}=\left\{\mathcal{M}, E_{M}\right\}$. Key to our perspective deformation process is the idea of using the structure of Delaunay graphs to find correspondences between the two point-sets. This correspondence matching is denoted by the function $f: \mathcal{M} \rightarrow \mathcal{D}$ from the nodes of the datagraph to those of the model graph. According to this notation the statements $(i, j) \in f^{(n)}$ or equivalently $f^{(n)}(i)=j$ indicate that there is a match between the node $i \in \mathcal{D}$ of the model-graph to the node $j \in \mathcal{M}$ of the model graph at iteration $n$ of the algorithm.

We exploit the structure of the Delaunay graphs to compute the consistency of match using the Bayesian framework for relational graph-matching recently 
reported by Wilson and Hancock [12-14]. Details of the method are outside the scope of this paper. Suffice to say that consistency of a configuration of matches residing on the neighbourhood $R_{i}=i \cup\left\{k ;(i, k) \in E_{D}\right\}$ of the node $i$ in the data-graph and its counterpart $S_{j}=j \cup\left\{l ;(j, l) \in E_{m}\right\}$ for the node $j$ in the model-graph is gauged by Hamming distance. The Hamming distance $H(i, j)$ counts the number of matches on the data-graph neighbourhood $R_{i}$ that are inconsistently matched onto the model-graph neighbourhood $S_{j}$. According to Wilson and Hancock [12-14] the probability that the data-graph node $i$ matches to the model-graph node $j$ at iteration $n$ of the algorithm is given by

$$
\zeta_{i, j}^{(n)}=\frac{\exp [-\beta H(i, j)]}{\sum_{j \in \mathcal{M}} \exp [-\beta H(i, j)]}
$$

In the above expression, the Hamming distance is given by

$$
H(i, j)=\sum_{\left(u_{k}, v_{k}\right) \in R_{2} \bullet S_{j}}\left(1-\delta_{f^{(n)}\left(u_{k}\right), v_{k}}\right)
$$

where the symbol - denotes the composition of the data-graph relation $R_{i}$ and the model-graph relation $S_{j}$. The exponential constant $\beta=\ln \frac{1-P_{e}}{P_{e}}$ is related to the uniform probability of structural matching errors $P_{e}$. This probability is set to reflect the overlap of the two point-sets In the work reported here we set $P_{e}$ as follows

$$
P_{e}=\frac{2|| \mathcal{M}|-| \mathcal{D}||}{\| \mathcal{M}|+| \mathcal{D}||}
$$

\section{The EM Algorithm}

In this section we describe the main stages of our iterative matching scheme. Our aim is to extract both maximum likelihood perspective parameters and maximum a posteriori matching probabilities using coupled update operations. In the spirit of Dempster, Laird and Rubin's EM algorithm [4], we aim to condition the updated parameter estimates (i.e. $\Phi^{(n+1)}$ ) on the most recently available correspondence matches (i.e. $\left.f^{(n)}\right)$. In other words, the maximum-likelihood parameters satisfy the following condition

$$
\Phi^{(n+1)}=\arg \max _{\Phi} p\left(\Phi \mid \mathbf{w}, f^{(n)}\right)
$$

In a similar way, the maximum a posteriori matches are conditioned upon the most recently available parameter-estimates. The matching configuration therefore satisfies the following condition

$$
f^{(n+1)}=\arg \max _{f} P\left(f \mid \mathbf{w}, \Phi^{(n)}\right)
$$




\subsection{Expectation}

We have recently shown how coupled updates of this form can be realised through the optimisation of single integrated expected likelihood function. Details of the formal development are outside the scope of this paper and can found in the recent account of Turner and Hancock [10]. Suffice to say that the parameters and the correspondence matches may be sought through joint optimisation of the quantity

$$
Q\left(\Phi^{(n+1)} \mid \Phi^{(n)}\right)=\sum_{(i, j) \in f^{(n)}} P\left(\underline{\underline{z}}_{j} \mid \underline{w}_{i}, \Phi^{(n)}\right) \zeta_{i, j}^{(n)} \ln p\left(\underline{w}_{i} \mid \underline{\underline{z}}_{j}, \Phi^{(n+1)}\right)
$$

The structure of this expected log-likelihood function requires further comment. The measurement densities $p\left(\underline{\mathrm{w}}_{i} \mid \underline{\mathrm{z}}_{j}, \Phi^{(n+1)}\right)$ model the distribution of errorresiduals between the observed model-point position $\underline{w}_{i}$ and the predicted position of the model point $\underline{z}_{j}$ under the current set of transformation parameter $\Phi^{(n+1)}$. The log-likelihood contributions at iteration $n+1$ are weighted by the $a$ posteriori measurement probabilities $P\left(\underline{\mathrm{z}}_{j} \mid \underline{\mathrm{w}}_{i}, \Phi^{(n)}\right)$ computed at iteration $n$ of the algorithm. Following Jordan and Jacobs [5] we gate the individual expectedlikelihood contributions using the the structural matching probabilities $\zeta_{i, j}^{(n)}$. Finally, the summation extends over the set of correspondence matches $(i, j) \in f^{(n)}$ available at iteration $n$.

Using the Bayes rule, we can re-write the a posteriori measurement probabilities in terms of the of the conditional measurement densities

$$
P\left(\underline{\mathrm{z}}_{j} \mid \underline{\mathrm{w}}_{i}, \Phi^{(n)}\right)=\frac{\alpha_{j}^{(n)} p\left(\underline{\mathrm{w}}_{i} \mid \underline{\mathrm{z}}_{j}, \Phi^{(n)}\right)}{\sum_{j^{\prime} \in \mathcal{M}} \alpha_{j^{\prime}}^{(n)} p\left(\underline{\mathrm{w}}_{i} \mid \underline{\mathrm{z}}_{j^{\prime}}, \Phi^{(n)}\right)}
$$

The mixing proportions are computed by averaging the a posteriori probabilities over the set of data-points, i.e.

$$
\alpha_{j}^{(n+1)}=\frac{1}{|\mathcal{D}|} \sum_{i \in \mathcal{D}} P\left(\underline{z}_{j} \mid \underline{w}_{i}, \Phi^{(n)}\right)
$$

\subsection{Gaussian Error Model}

In order to proceed with the development of a point registration process we require a model for the conditional measurement densities, i.e. $p\left(\underline{w}_{i} \mid \underline{z}_{j}, \Phi^{(n)}\right)$. Here we assume that the required model can be specified in terms of a multivariate Gaussian distribution. The random variables appearing in these distributions are the error residuals for the position predictions of the $j$ th model point delivered by the current estimated transformation parameters. Accordingly we write

$$
p\left(\underline{\mathrm{w}}_{i} \mid \underline{\underline{z}}_{j}, \Phi^{(n)}\right)=\frac{1}{(2 \pi)^{\frac{3}{2}} \sqrt{|\Sigma|}} \exp \left[-\frac{1}{2} \epsilon_{i, j}\left(\Phi^{(n)}\right)^{T} \Sigma^{-1} \epsilon_{i, j}\left(\Phi^{(n)}\right)\right]
$$


In the above expression $\Sigma$ is the variance-covariance matrix for the vector of error-residuals $\epsilon_{i, j}\left(\Phi^{(n)}\right)=\underline{\mathrm{w}}_{i}-\underline{\mathrm{z}}_{j}^{(n)}$ between the components of the predicted measurement vectors $\mathrm{z}_{j}^{(n)}$ and their counterparts in the data, i.e. $\mathbf{w}_{i}$. Formally, the matrix is related to the expectation of the outer-product of the error-residuals i.e. $\Sigma=E\left[\epsilon_{i, j}\left(\Phi^{(n)}\right) \epsilon_{i, j}\left(\Phi^{(n)}\right)^{T}\right]$.

With these ingredients, the expectation step of the EM algorithm simply reduces to computing the weighted squared error criterion

$$
Q^{\prime}\left(\Phi^{(n+1)} \mid \Phi^{(n)}\right)=\sum_{(i, j) \in f^{(n)}} P\left(\underline{\mathrm{z}}_{j} \mid \underline{\mathrm{w}}_{i}, \Phi^{(n)}\right) \zeta_{i, j}^{(n)} \epsilon_{i, j}\left(\Phi^{(n+1)}\right)^{T} \Sigma^{-1} \epsilon_{i, j}\left(\Phi^{(n+1)}\right)
$$

In other words, the a posteriori probabilities $P\left(\underline{z}_{j} \mid \underline{w}_{i}, \Phi^{(n)}\right)$ and the structural matching probabilities $\zeta_{i, j}^{(n)}$ effectively regulate the contributions to the likelihood function. Matches for which there is little evidence contribute insignificantly, while those which are in good registration dominate.

\subsection{Maximisation}

The maximisation step of our perspective deformation algorithm is based on dual coupled update processes. The first of these aims to locate maximum $a$ posteriori probability correspondence matches. The second update operation is concerned with locating maximum likelihood perspective parameters. We effect the coupling by allowing information flow between the two processes.

Maximum a posteriori probability matches Point correspondences are sought so as to maximise the a posteriori probability of structural match. Individual point-correspondences should be updated in the following manner

$$
f^{(n+1)}(i)=\arg \max _{j \in \mathcal{M}} P\left(\underline{\mathrm{z}}_{j} \mid \underline{\mathrm{w}}_{i}, \Phi^{(n)}\right) \zeta_{i, j}^{(n)}
$$

Once this update equation has been applied, the unmatched model-graph nodes are identified for removal from the triangulation. At this point the edited set of model feature-points is re-triangulated along the lines suggested in Section 3 The updated structural matching probabilities $\zeta_{i, j}^{(n+1)}$ are also updated using equations (3) and (4) as outlined in Section 2.

Maximum likelihood parameters Maximising the expected log-likelihood function is equivalent to minimising the following weighted squared error criterion

$$
\Phi^{(n+1)}=\arg \min _{\Phi} \sum_{(i, j) \in f^{(n)}} P\left(\underline{z}_{j} \mid w_{i}, \Phi^{(n)}\right) \zeta_{i, j}^{(n)} \epsilon_{i, j}(\Phi)^{T} \Sigma^{-1} \epsilon_{i, j}(\Phi)
$$




\section{Experiments}

The real-world evaluation of our matching method is concerned with recognising planer objects in different 3D poses. The object used in this study is a 3.5 inch floppy disk which is placed on a desktop. The scene is viewed with a lowquality SGI IndyCam. The feature points used to triangulate the object are corners. Since the imaging process is not accurately modelled by a perspective transformation under pin-hole optics, the example provides a challenging test of our matching process.

Our experiments are illustrated in Figure 1. The first two columns show the views under match. In the first example (the upper row of Figure 1) we are concerned with matching when there is a significant difference in perspective forshortening. In the example shown in the second row of Figure 1, there is a rotation of the object in addition to the foreshortening. The final two rows show the matching of a fronto-parallel view against rotated and forshortened ones. The images in the third column are the initial matching configurations. Here the perspective parameter matrix has been selected at random. The fourth column in Figure 1 shows the final matching configuration after the EM algorithm has converged. In all four cases the final registration is accurate. The algorithm appears to be capable of recovering good matches even when the initial pose estimate is poor.

\section{Conclusions}

Our main contribution in this paper has been to develop a new algorithm for matching under perspective deformation. The method integrates both relational point-structure and point-distribution information into the deformation process. Matching is realised using the EM algorithm. We have illustrated the effectiveness of the resulting perspective deformation process in the matching of $3 \mathrm{D}$ poses of planar objects.

\section{References}

1. N Ahuja, "Dot Pattern Processing using Voronoi Neighbourhoods", IEEE PAMI, 4, pp 336-343, 1982.

2. J-D. Boissonnat, "Geometric Structures for Three-Dimensional Shape Representation", ACM Transactions on Graphics, 3, pp. 266-286, 1984.

3. T.F Cootes, C.J. Taylor, D.H. Cooper and J. Graham, "Active Shape Models - Their Training and Application", Computer Vision, Graphics and Image Understanding, 61, pp. 38-59, 1995.

4. A.P. Dempster, Rubin N.M. and Rubin D.B., "Maximum-likelihood from incomplete data via the EM algorithm", J. Royal Statistical Soc. Ser. B (methodological),39, pp 1-38, 1977.

5. M.I. Jordan and R.A. Jacobs, "Hierarchical Mixtures of Experts and the EM Algorithm", Neural Computation, 6, pp. 181-214, 1994. 

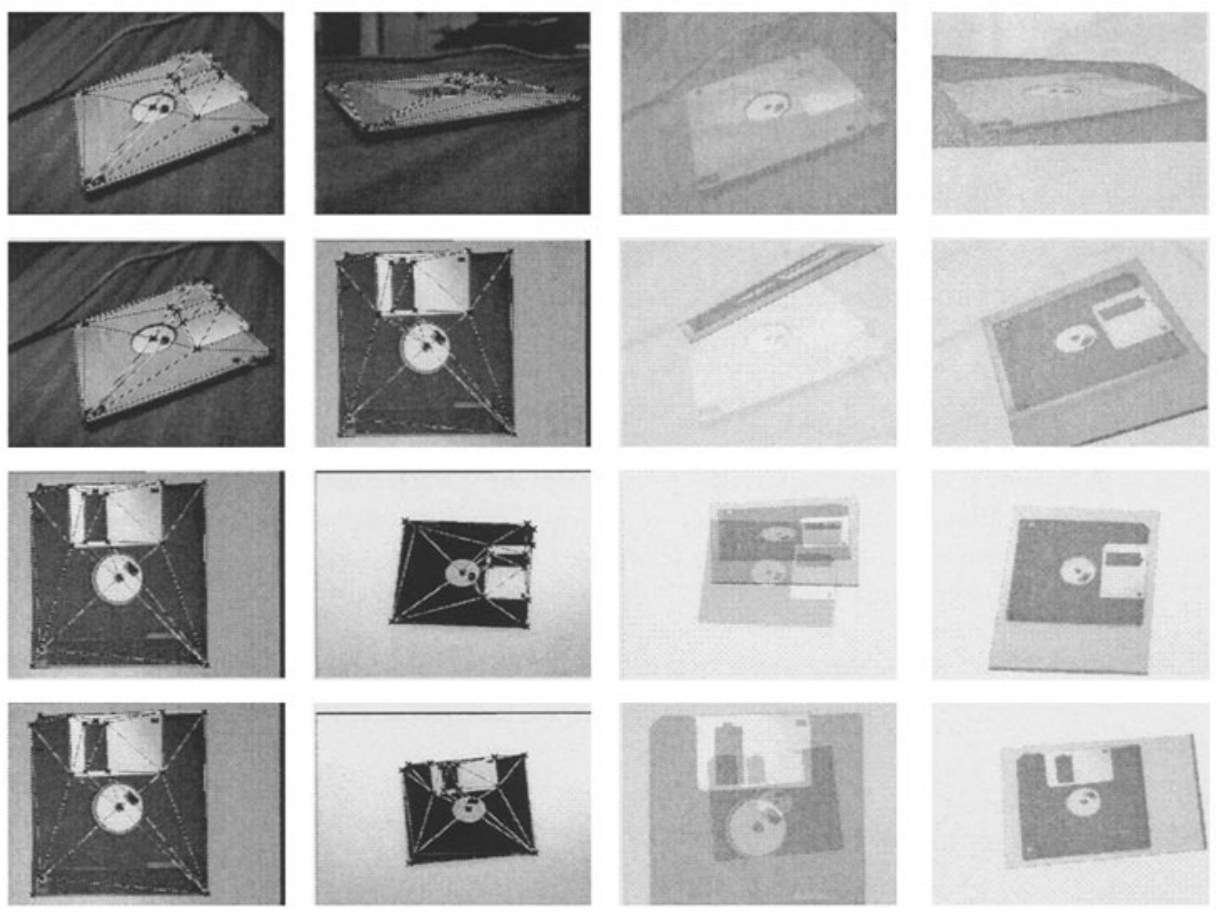

Fig. 1. Left to right : model, data, initial configurations and final configuration for the matching process.

6. D.P. McReynolds and D.G. Lowe, "Rigidity Checking of 3D Point Correspondences under Perspective Projection", IEEE PAMI, 18, pp. 1174-1185, 1996.

7. G. L. Scott and H.C. Longuet-Higgins, "An Algorithm for Associating the Features of 2 Images", Proceedings of the Royal Society of London Series B-Biological, 244, pp. $21-26,1991$.

8. S. Sclaroff and A.P. Pentland, "Modal Matching for Correspondence and Recognition", IEEE PAMI, 17, pp. 545-661, 1995.

9. L.S. Shapiro and J. M. Brady, "Feature-based Correspondence - An Eigenvector Approach", Image and Vision Computing, 10, pp. 283-288, 1992.

10. M. Turner and E.R. Hancock, "An EM-like Relaxation Operator", Proceedings of the Thirteenth International Conference on Pattern Recognition, Volume II, pp. $166-170,1996$.

11. S. Ullman, "The Interpretation of Visual Motion", MIT Press, 1979.

12. R.C. Wilson, A.N. Evans and E.R. Hancock, "Relational Matching by Discrete Relaxation", Image and Vision Computing, 13, pp. 411-421, 1995.

13. R.C. Wilson and E.R. Hancock, "Relational Matching with Dynamic Graph Structures", Proceedings of the Fifth International Conference on Computer Vision, pp. 450-456, 19

14. R.C. Wilson and E.R. Hancock, "Gauging Relational Consistency and Correcting Structural Errors", IEEE Computer Society Computer Vision and Pattern Recognition Conference, pp. 54-62, 1996. 